\title{
Endoscopic features of gastrointestinal stromal tumor in the small intestine
}

\author{
Yutaro Ihara ${ }^{1}$, Takehiro Torisu ${ }^{1}$, Tomohiko Moriyama ${ }^{1}$, Junji Umeno ${ }^{1}$, Atsushi Hirano ${ }^{1}$, Yasuharu Okamoto ${ }^{1}$, \\ Yoshifumi Hori ${ }^{2}$, Hidetaka Yamamoto ${ }^{2}$, Takanari Kitazono ${ }^{1}$, Motohiro Esaki ${ }^{1,3}$ \\ Departments of ${ }^{1}$ Medicine and Clinical Science, and ${ }^{2}$ Anatomic Pathology, Graduate School of Medical Sciences, Kyushu University, Fukuoka; \\ ${ }^{3}$ Department of Endoscopic Diagnostics and Therapeutics, Saga University Hospital, Saga, Japan
}

Background/Aims: Gastrointestinal stromal tumor (GIST) is one of the most common types of submucosal tumors (SMTs). Because of GIST's malignant potential, it is crucial to differentiate it from other SMTs. The present study aimed to identify characteristic endoscopic findings of GISTs in the small intestine. Methods: We reviewed the clinicopathological and endoscopic findings of 38 patients with endoscopically or surgically resected SMTs in the small intestine. SMTs were classified into GIST and non-GIST groups, and clinicopathological and endoscopic findings were compared between the 2 groups. Results: Fifteen patients had GIST and 23 patients had other types of SMTs in the small intestine. Comparison of the endoscopic findings between the 2 groups revealed that dilated vessels in the surrounding mucosa were significantly more in number in the GIST group than in the non-GIST group $(P<0.05)$. However, there were no other differences in endoscopic findings between the 2 groups. Among patients with GISTs, the presence of dilated vessels in the surrounding mucosa was not associated with bleeding risk, tumor size, or metastasis rate at diagnosis. Conclusions: Dilated vessels in the surrounding mucosa, identified during balloon-assisted endoscopy, may be a diagnostic indicator for GIST in the small intestine. However, its clinical significance should be further analyzed. (Intest Res 2019;17:398-403)

Key Words: Balloon-assisted enteroscopy; Dilated vessel; Gastrointestinal stromal tumors; Intestine, small

\section{INTRODUCTION}

Gastrointestinal stromal tumor (GISTs) is a mesenchymal tumor of the GI tract, with the small intestine being the second most frequent location, accounting for $20 \%-30 \%$ of all GISTs. ${ }^{1-3}$ The biological behaviors of GIST vary widely, although it is generally considered to have malignant potential, with GISTs in the small intestine considered to manifest more aggressive clinical behavior compared with gastric GISTs. ${ }^{4,5}$ However, this difference in clinical behaviors might be partly attributed to

Received December 12, 2018. Revised April 11, 2019. Accepted April 19, 2019. Correspondence to Motohiro Esaki, Department of Medicine and Clinical Science, Graduate School of Medical Sciences, Kyushu University, Maidashi 3-1-1, Higashi-ku, Fukuoka 812-8582, Japan. Tel: +81-92-642-5261, Fax: +81-92-642-5273, E-mail: mesaki@intmed2.med.kyushu-u.ac.jp ORCID Yutaro Ihara (https://orcid.org/0000-0003-0430-7061), Motohiro Esaki (https://orcid.org/0000-0002-1195-0074) the different accessibilities of the tumors, with gastric GISTs sometimes detected incidentally by esophagogastroduodenoscopy, while most small intestinal GISTs are only detected following obstruction or bleeding symptoms. Small intestinal GISTs have accordingly been reported to be larger than gastric GISTs at the time of diagnosis. ${ }^{6}$

Recent developments in balloon-assisted endoscopy (BAE) and capsule endoscopy have enabled the endoscopic observation of small intestinal pathologies. BAE is particularly useful for diagnosing tumorous lesions in the small intestine by allowing precise observation and bioptic examination. However, it is often difficult to make a pathological diagnosis of submucosal tumor (SMT) by bioptic examination under BAE. ${ }^{7.8}$ Although endoscopic ultrasonography (EUS) and fine-needle aspiration can be used to diagnose SMT, this procedure may be difficult to perform in the small intestine. ${ }^{9,10}$ It is thus con- 
sidered important to be aware of the characteristic endoscopic findings of small intestinal SMTs, especially malignant SMTs such as GIST. ${ }^{11}$ In the present study, we compared the endoscopic findings between GIST and other SMTs to identify potential diagnostic clue of small intestinal GIST under BAE.

\section{METHODS}

\section{Patients}

This study included 38 cases with small intestinal SMTs found by BAE and endoscopically or surgically resected between September 2004 and October 2018. One case was treated by endoscopic mucosal resection and 37 cases underwent surgical resection. The clinical data of each patient were obtained by review of patient records at Kyushu University Hospital, and included information on gender, age, clinical symptoms, and laboratory data, including hemoglobin, lactate, and dehydrogenase at the time of BAE. Contrast-enhanced CT (CE-CT) findings were also reviewed for patients with small intestinal GISTs. Histopathological diagnosis of the small intestinal SMT was confirmed in all cases, and it was determined by 3 pathologists (Y.I., M.E., and H.Y.) based on H\&E staining and immunohistochemical staining, as required.

The study protocol was approved by the ethics committee at Kyushu University Hospital (IRB No. 30-485), and the study was conducted in accordance with the Helsinki Declaration. Informed consent was obtained from all patients.

\section{Double-Balloon Endoscopy}

BAE was performed using a Double-Balloon Endoscopy System (Fujinon-Toshiba ES System Co., Tokyo, Japan). Antegrade and retrograde BAE were performed as described previously. ${ }^{12}$ The BAE route was determined by small bowel barium examination, capsule endoscopy, or abdominal CT, while some patients underwent both antegrade and retrograde BAE.

The BAE images were retrospectively reviewed by 2 expert endoscopists (Y.I. and T.T.). The following endoscopic findings were determined: color of the tumor surface (yellowish, bluish, reddish, or same as the surrounding mucosa), nodularity of the tumor (smooth or multinodular), presence of ulcer (none, single, or multiple), and abnormality of the surrounding mucosa (dilated vessels). Disagreements regarding the endoscopic findings were resolved by discussion between the 2 endoscopists. Because endoscopic determination can underestimate the tumor size of small bowel SMTs, the tumor size was determined based on the endoscopically or surgically resected specimens.

\section{Statistical Analysis}

Categorical variables were described as frequencies and numerical variables as median (range). Clinicopathological and endoscopic findings were compared between the groups using Fisher exact probability test or Wilcoxon signed-rank test, as appropriate. All statistical analyses were performed using JMP Proll (SAS Institute Inc., Cary, NC, USA). A $P$-value $<0.05$ was considered statistically significant.

\section{RESULTS}

\section{Demographic Data}

Of the 38 patients, 15 were diagnosed with GIST (GIST group) and the remaining 23 patients were diagnosed with small intestinal SMT other than GIST (non-GIST group). In the nonGIST group, malignant lymphoma manifesting SMT like mass was found in 16 patients, neuroendocrine tumors in 2 patients, heterotopic pancreas in 2 patients, leiomyosarcoma in 1 patient, dedifferentiated liposarcoma in 1 patient, and metastatic tumor in 1 patient. Histological types of malignant lymphoma included follicular lymphoma $(\mathrm{n}=5)$, mucosa-associated lymphoid tissue lymphoma $(\mathrm{n}=2)$, diffuse large B-cell lymphoma $(\mathrm{n}=5)$, Burkitt lymphoma $(\mathrm{n}=1)$ or T-cell lymphoma $(\mathrm{n}=3)$. The clinical characteristics of the GIST and non-GIST groups are compared in Table 1. There were no significant differences in age, gender, clinical symptoms, or laboratory data at the time of BAE between the 2 groups.

\section{Endoscopic Findings in GIST and Non-GIST Groups}

Twenty-four of the 38 patients underwent antegrade BAE, 12 underwent retrograde $\mathrm{BAE}$, and the remaining 2 patients underwent both antegrade and retrograde BAE. GISTs were found in the jejunum in 12 patients and the ileum in 3 patients, and small intestinal non-GISTs were located in the duodenum in 1 , the jejunum in 13, and the ileum in 9 patients. The sites of the lesions were not significantly different in the 2 groups $(P=0.292)$.

The endoscopic findings in the GIST and non-GIST groups are compared in Table 2. The tumor size, color of the tumor surface, nodularity of the tumors, and the presence of ulcers were not different in both groups, but dilated vessels in the surrounding mucosa were significantly more frequently found in the GIST compared with the non-GIST group $(P<0.001)$. Two representative cases with dilated vessels in the surrounding mucosa are shown in Fig. 1. Representative images of SMT other than GIST were shown in Fig. 2. 
Table 1. Clinical Features of Patients with GISTs and Non-GIST SMTs in the Small Intestine

\begin{tabular}{|c|c|c|c|}
\hline Characteristic & $\begin{array}{l}\text { GIST group } \\
(n=15)\end{array}$ & $\begin{array}{c}\text { Non-GIST } \\
\text { group }(n=23)\end{array}$ & $P$-value \\
\hline Sex (female/male) & $6 / 9$ & $13 / 10$ & 0.508 \\
\hline Age (yr) & $65(40-82)$ & $66(34-80)$ & 0.846 \\
\hline \multicolumn{4}{|l|}{ Symptoms } \\
\hline Abdominal pain & 5 & 10 & 0.736 \\
\hline Abdominal distension & 4 & 5 & 1.000 \\
\hline Hematemesis or melena & 4 & 5 & 0.451 \\
\hline None & 6 & 7 & 0.728 \\
\hline Hemoglobin (g/dL) & $12.6(6.3-16.1)$ & $12.2(8.1-15.6)$ & 0.487 \\
\hline $\mathrm{LDH}(\mathrm{g} / \mathrm{dL})$ & $190(141-411)$ & $194(128-529)$ & 0.811 \\
\hline \multicolumn{4}{|l|}{ Pathology } \\
\hline GIST & 15 & & \\
\hline Malignant lymphoma & & 16 & \\
\hline Follicular & & 5 & \\
\hline MALT & & 2 & \\
\hline DLBCL & & 5 & \\
\hline Burkitt & & 1 & \\
\hline T-cell & & 3 & \\
\hline Neuroendocrine tumors & & 2 & \\
\hline Heterotopic pancreas & & 2 & \\
\hline Leiomyosarcoma & & 1 & \\
\hline Dedifferentiated liposarco & & 1 & \\
\hline Metastasis tumor & & 1 & \\
\hline
\end{tabular}

Values are presented as number or median (range).

GIST, GI stromal tumor; SMT, submucosal tumor; MALT, mucosa-associated lymphoid tissue; DLBCL, diffuse large B-cell lymphoma.

\section{Clinicopathological Features in GIST Patients with and without Dilated Vessels}

There was no significant difference in the incidence of hematemesis or melena between GIST patients with and without dilated vessels. There was also no difference in median hemoglobin values between GIST patients with dilated vessels (13.2

Table 2. Endoscopic Findings in Patients with GISTs and NonGIST SMTs in the Small Intestine

\begin{tabular}{lccc}
\hline Characteristic & $\begin{array}{c}\text { GIST group } \\
(\mathrm{n}=15)\end{array}$ & $\begin{array}{c}\text { Non-GIST } \\
\text { group } \\
(\mathrm{n}=23)\end{array}$ & P-value \\
\hline Tumor size (mm) & $32(22-95)$ & $60(5-300)$ & 0.193 \\
Color & 0 & 1 & 1.000 \\
$\quad$ Yellowish & 1 & 0 & 0.395 \\
$\quad$ Bluish & 0 & 5 & 0.137 \\
$\quad$ Reddish & 14 & 17 & 0.209 \\
$\quad$ Similar to the surrounding mucosa & & & 0.508 \\
$\quad$ Nodularity & 9 & 10 & \\
$\quad$ Smooth protrusion & 6 & 13 & \\
$\quad$ Multinodular protrusion & & & 0.453 \\
$\quad$ Ulcer & 8 & 8 & \\
$\quad$ None & 6 & 14 & \\
$\quad$ Single & 1 & 1 & \\
$\quad$ Multiple & & & \\
$\quad$ Surrounding mucosa & & \\
$\quad$ Dilated vessels & 7 & 0 & $<0.001$ \\
\hline
\end{tabular}

Values are presented as number or median (range). GIST, GI stromal tumor; SMT, submucosal tumor.
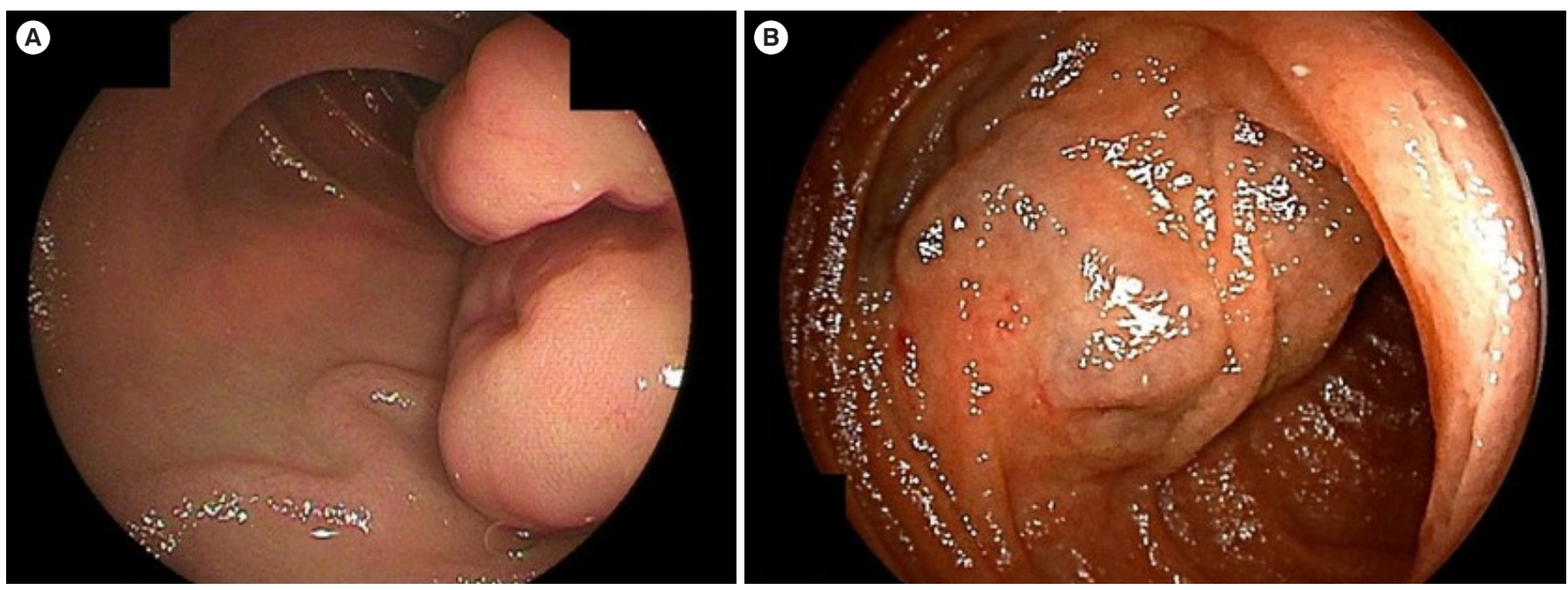

Fig. 1. Double-balloon endoscopy images of gastrointestinal stromal tumor. (A) Multinodular submucosal tumor with tortuous dilated vessels. (B) Blue dilated vessels on the surface of the tumor and surrounding mucosa. 

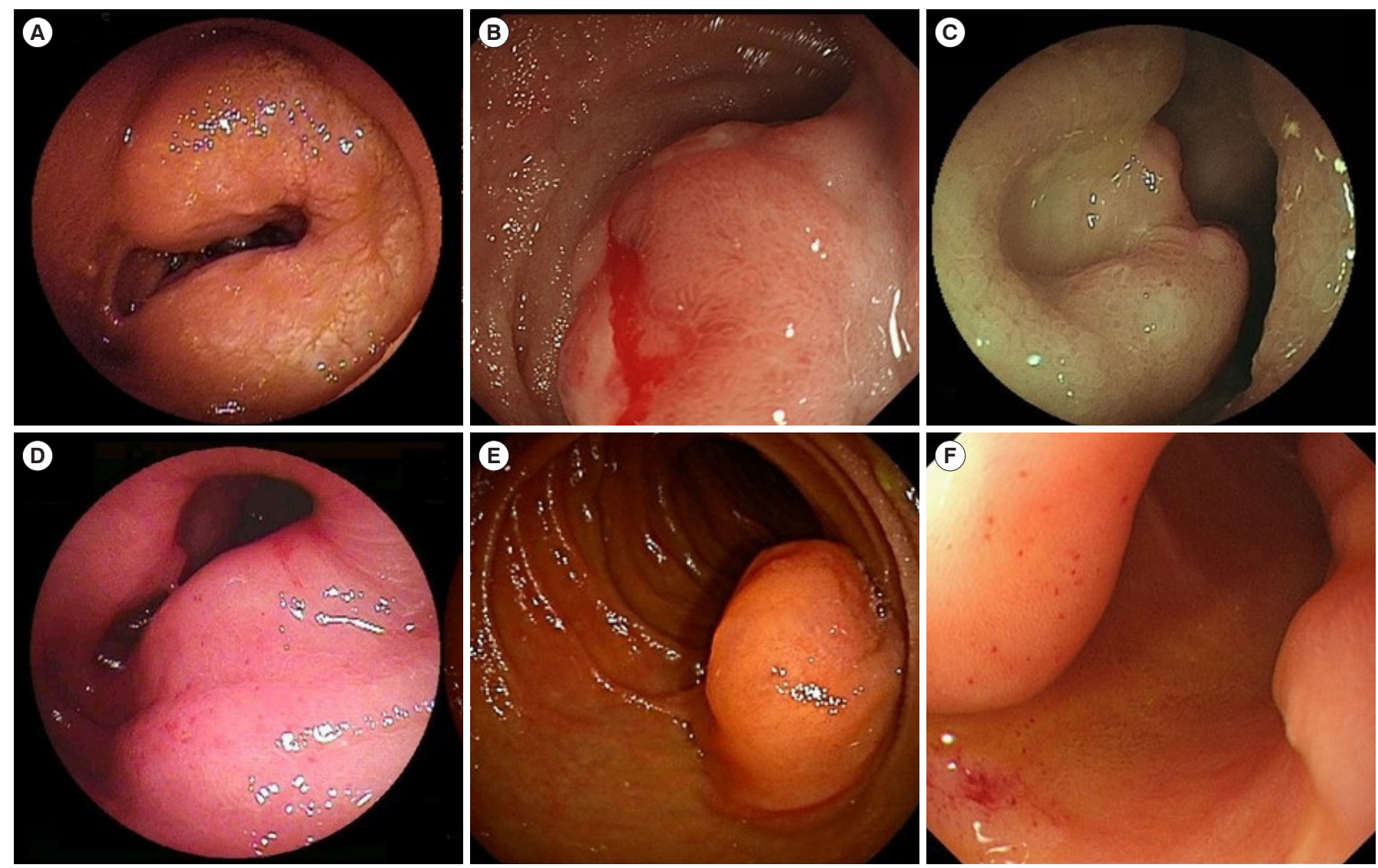

Fig. 2. Representative images of submucosal tumor other than gastrointestinal stromal tumor. (A) Follicular lymphoma. (B) Mucosa-associated lymphoid tissue lymphoma. (C) Diffuse large B-cell lymphoma. (D) T-cell lymphoma. (E) Heterotopic pancreas. (F) Dedifferentiated liposarcoma.

$\mathrm{g} / \mathrm{dL}$ [range, 6.3-16.1 g/dL]) and those without (12.4 g/dL [range, 8.9-14.7 g/dL]). However, one patient with GIST accompanied by dilated vessels developed hemorrhagic shock after bioptic examination under double-balloon endoscopy.

The histological features of GISTs with and without dilated vessels are compared in Table 3. Tumor size, tumor growth pattern, risk grade by Miettinen classification, ${ }^{4}$ and distant metastasis were no different in both groups. We also compared tumor enhancement patterns in the 14 patients who underwent CECT. However, 11 GISTs showed homogenous enhancement, resulting in no significant difference in CE-CT findings according to the presence of dilated vessels ( $86 \%$ vs. $71 \%, P=0.559$ ).

\section{DISCUSSION}

Histological confirmation is crucial for making treatment decisions in patients with GI tumorous lesions, particularly in the case of malignant tumors such as GIST. However, it may be difficult to obtain tumor biopsies from GISTs because of their configuration. The rate of histological diagnosis of GIST by enteroscopy was previously reported to be only $40 \%-45 \%$, ${ }^{7,8}$ and was only $46.7 \%$ even in cases of small bowel GIST with ulceration. ${ }^{11}$ Similarly, bioptic examination under enteroscopy only confirmed a histological diagnosis of GIST in $33 \%$ of the current cases. We therefore aimed to identify any endoscopic findings that could help differentiate between GISTs and other SMTs in the small intestine.

We only recruited cases with small intestinal SMTs that had been endoscopically or surgically resected in the present study. This is because precise determination of tumor size is indispensable for the analysis of endoscopic findings of small intestinal SMTs, and because endoscopy can underestimate the tumor size when the tumor shows an eccentric extraluminal growth pattern. Consequently, non-GIST group was mostly composed of malignant lymphoma that shows endoscopic variation such as ulcerative, nodular (including SMT like mass), infiltrative or diminutive mucosal changes. Malignant lymphoma manifesting SMT like mass alone was included in the 
Table 3. Pathological Findings of GISTs with and without Dilated Vessels

\begin{tabular}{|c|c|c|c|}
\hline & $\begin{array}{c}\text { Dilated } \\
\text { vessels } \\
\text { group }(n=7)\end{array}$ & $\begin{array}{c}\text { Without } \\
\text { dilated vessels } \\
\text { group }(n=8)\end{array}$ & $P$-value \\
\hline Tumor size (mm) & $28(25-95)$ & $40(22-75)$ & 0.486 \\
\hline Tumor growth pattern & & & 0.405 \\
\hline Intramural & 1 & 3 & \\
\hline Extramural & 2 & 3 & \\
\hline Intraluminal & 4 & 2 & \\
\hline Mib-1 index ${ }^{a}$ & $4.27 \pm 4.15$ & $3.22 \pm 3.26$ & 0.771 \\
\hline Miettinen classification & & & 0.978 \\
\hline Low & 4 & 5 & \\
\hline Intermediate & 1 & 1 & \\
\hline High & 2 & 2 & \\
\hline Metastasis at diagnosis & 1 & 0 & 0.467 \\
\hline
\end{tabular}

Values are presented as median (range), number, or mean \pm SD.

${ }^{a}$ Mib-1 index was determined by observing 1,000 nuclei in areas of the section with the highest labelling rates.

GIST, GI stromal tumor.

non-GIST group considering the aim of the present study.

The present study revealed no differences in color, nodularity, size, or presence of ulcers on the tumor surface between GISTs and other SMTs. It thus can be considered that central umbilication, ulceration of the overlying mucosa, or multiple nodularity were not the diagnostic clues for differentiating GIST from other malignant SMTs including malignant lymphoma, neuroendocrine tumors, and metastatic tumor, while such findings are indicated as diagnostic hints for malignant SMTs. However, dilated vessels in the surrounding mucosa were significantly more frequent in GISTs than in other small intestinal SMTs, suggesting that this endoscopic findings might be a clue to a diagnosis of small intestinal GIST under BAE.

Fukuta et al. ${ }^{13}$ previously proposed a classification of GISTs according to blood flow demonstrated under contrast-enhanced harmonic EUS, while another study further indicated a possible association between the presence of intra-tumoral vessels under contrast-enhanced harmonic EUS and malignant potential in gastric GIST. ${ }^{14}$ Dilated vessels in the mucosa surrounding small intestinal GISTs might imply a rich tumor blood flow, indicating that this endoscopic findings may predict the malignant potential of small intestinal GISTs. However, the current study found no differences in histological or CE-CT findings between GISTs with and without dilated vessels. It thus can be possible that dilated vessels in the surrounding mucosa observed endoscopically do not necessarily indicate abundant vascularization of small intestinal GISTs. Furthermore, type 2 error caused by the small sample size might be attributed to the insignificant results. Further studies with larger sample size is warranted to determine the association of dilated vessels with malignant potential of small intestinal GISTs.

We also analyzed the clinical impact of the endoscopic findings by comparing clinical symptoms and the risk of bleeding caused by bioptic examination. Two previously reported cases presented with overt GI bleeding as the initial clinical symptom of small intestinal GISTs, ${ }^{15,16}$ while hemorrhagic complications were reported in $1.9 \%$ of patients who underwent bioptic examination under BAE. ${ }^{8}$ One patient in the present study experienced hemorrhagic shock after bioptic examination and another showed severe anemia before the diagnosis, both of whom had GISTs with dilated vessels in the surrounding mucosa. Although we found no significant difference in bleeding complications between patients with and without dilated vessels, it may be preferable to avoid bioptic examination in patients with small intestinal SMTs accompanied by dilated vessels in the surrounding mucosa identified by BAE.

The present study had several limitations. First, the number of subjects was small, which might have affected the statistical analysis. Second, it was a retrospective study with limited clinical data, and the possibility of selection bias cannot be excluded. Furthermore, it was difficult to evaluate the histological findings of small intestinal SMTs in relation to the surrounding mucosa, including the presence of dilated vessels.

In conclusion, the presence of dilated vessels in the surrounding mucosa may be an endoscopic clue for differentiating between GIST and other SMTs in the small intestine. Further studies with larger numbers of subjects with a variety of small bowel SMTs are needed to confirm the clinical impact of this endoscopic finding in small intestinal GISTs.

\section{FINANCIAL SUPPORT}

The authors received no financial support for the research, authorship, and/or publication of this article.

\section{CONFLICT OF INTEREST}

No potential conflict of interest relevant to this article was reported. 


\section{AUTHOR CONTRIBUTION}

Original draft: Esaki M. Endoscopy: Torisu T, Moriyama T, Umeno J, Hirano A, Okamoto Y, Esaki M. Pathological diagnosis: Hori Y, Yamamoto H. Writing: Ihara Y. Analysis: Ihara Y. Review: Torisu T, Moriyama T, Umeno J, Hirano A, Okamoto Y, Kitazono T, Esaki M. Editing: Torisu T, Esaki M. Approval of final manuscript: all authors.

\section{REFERENCES}

1. Pidhorecky I, Cheney RT, Kraybill WG, Gibbs JF. Gastrointestinal stromal tumors: current diagnosis, biologic behavior, and management. Ann Surg Oncol 2000;7:705-712.

2. Tran T, Davila JA, El-Serag HB. The epidemiology of malignant gastrointestinal stromal tumors: an analysis of 1,458 cases from 1992 to 2000. Am J Gastroenterol 2005;100:162-168.

3. Miettinen M, Lasota J. Gastrointestinal stromal tumors: review on morphology, molecular pathology, prognosis, and differential diagnosis. Arch Pathol Lab Med 2006;130:1466-1478.

4. Miettinen M, Lasota J. Gastrointestinal stromal tumors: pathology and prognosis at different sites. Semin Diagn Pathol 2006;23:70-83.

5. Dematteo RP, Gold JS, Saran L, et al. Tumor mitotic rate, size, and location independently predict recurrence after resection of primary gastrointestinal stromal tumor (GIST). Cancer 2008;112:608-615.

6. Giuliano K, Nagarajan N, Canner J, et al. Gastric and small intestine gastrointestinal stromal tumors: do outcomes differ? J Surg Oncol 2017;115:351-357.

7. Nakatani M, Fujiwara Y, Nagami Y, et al. The usefulness of double-balloon enteroscopy in gastrointestinal stromal tumors of the small bowel with obscure gastrointestinal bleeding. Intern Med 2012;51:2675-2682.
8. Mitsui K, Tanaka S, Yamamoto H, et al. Role of double-balloon endoscopy in the diagnosis of small-bowel tumors: the first Japanese multicenter study. Gastrointest Endosc 2009;70:498504.

9. Ito H, Inoue H, Ryozawa S, et al. Fine-needle aspiration biopsy and endoscopic ultrasound for pretreatment pathological diagnosis of gastric gastrointestinal stromal tumors. Gastroenterol Res Pract 2012;2012:139083

10. Sekine M, Imaoka H, Mizuno N, et al. Clinical course of gastrointestinal stromal tumor diagnosed by endoscopic ultrasound-guided fine-needle aspiration. Dig Endosc 2015;27:4452.

11. Nakano A, Nakamura M, Watanabe O, et al. Endoscopic characteristics, risk grade, and prognostic prediction in gastrointestinal stromal tumors of the small bowel. Digestion 2017;95: 122-131.

12. Yamamoto H, Sekine Y, Sato Y, et al. Total enteroscopy with a nonsurgical steerable double-balloon method. Gastrointest Endosc 2001;53:216-220.

13. Fukuta N, Kitano M, Maekawa K, Chikugo T, Kudo M. Estimation of the malignant potential of gastrointestinal stromal tumors: the value of contrast-enhanced coded phase-inversion harmonics US. J Gastroenterol 2005;40:247-255.

14. Sakamoto H, Kitano M, Matsui S, et al. Estimation of malignant potential of GI stromal tumors by contrast-enhanced harmonic EUS (with videos). Gastrointest Endosc 2011;73: 227-237.

15. Tomita T, Palacherla J, Zuckerman M, Dougherty S, Ghaleb M. Gastrointestinal stromal tumor of jejunum with angiodysplasia. Dig Dis Sci 2004;49:667-671.

16. Bardell T, Hookey LC, Jalink D, Hurlbut DJ, Paterson WG. GI stromal tumor unmasked by argon plasma coagulation of vascular ectasia. Gastrointest Endosc 2004;59:920-923. 